\title{
Development of a Method for Assessing Country-Based Flood Risk at the Global Scale
}

\author{
Yoshiyuki Imamura ${ }^{1}$
}

Accepted: 4 December 2021/Published online: 10 February 2022

(C) The Author(s) 2022

\begin{abstract}
International frameworks such as the Sustainable Development Goals and the Sendai Framework for Disaster Risk Reduction 2015-2030 require the quantification of country-based flood risk. However, few approaches at the global scale include the three necessary components (hazard, exposure, and vulnerability) for determining disaster risk and are country-based assessments, owing to major challenges such as limited data availability and vulnerability proxy selection. Therefore, in this study, a method was developed with the following features: Incorporating the hazard, exposure, and vulnerability components; Applicable to the vast majority of countries in the world; Visualizing priority countries and illustrating effective measures and strategies; Clear and easy to understand by leaders and decision makers of international organizations, governments, and other stakeholders; Identifying each country's challenges and providing guidance on specific issues for more detailed investigation and policy creation; Including more extensive factors compared with past studies. In Asia and the Pacific, the Flood Risk Index computed by the developed method is compared with the fatality ratio, and the results show that improving flood resilience secures people and society regardless of the magnitude and frequency of floods. Analysis at the global scale visualizes regional tendencies and indicates that countries closer to the equator have higher flood risk. Analysis of country-based flood risk based on five indicators demonstrates that the developed method can assist international organizations, governments, and other
\end{abstract}

Yoshiyuki Imamura

imamura@tmu.ac.jp

1 Department of Civil and Environmental Engineering, Tokyo Metropolitan University, 192-0397 Hachioji, Tokyo, Japan stakeholders to further examine country-specific conditions and establish and implement policies and strategies toward building a resilient society and achieving international targets.

Keywords Asia and the Pacific - Country-based analysis · Flood risk index · Global scale · Vulnerability proxy

\section{Introduction}

Target 11.5 of the Sustainable Development Goals (SDGs) is "by 2030, significantly reduce the number of deaths and the number of people affected and substantially decrease the direct economic losses relative to global gross domestic product caused by disasters, including water-related disasters, with a focus on protecting the poor and people in vulnerable situations" (United Nations 2015, p. 22), which highlights the importance of water-related disasters. Among water-related disasters, floods are the most common, accounting for $43 \%$ of all natural hazard-related disasters from 1995 to 2015 (CRED and UNISDR 2015).

The Sendai Framework for Disaster Risk Reduction 2015-2030 (SFDRR) sets "understanding disaster risk" as its first priority for action (UNISDR 2015). The previous global framework, the Hyogo Framework for Action 2005-2015 adopted in 2005, recommended the development of "systems of indicators of disaster risk and vulnerability at national and subnational scales that will enable decision-makers to assess the impact of disasters" as a key activity (UNISDR 2007, p. 7). Flood risk assessment at the global scale is needed for international financing institutes to develop risk profiles and decide which risk reduction schemes to invest in (UNISDR 2007). Furthermore, the 
Asian Water Development Outlook 2013, issued by the Asian Development Bank, states that "the country-based findings, rankings, and key messages in the report indicate directions and priorities for increased investment, improved governance, and expanded capacity building" (ADB and APWF 2013, p. vi-2). Therefore, quantitative measurements of country-based flood risk would assist national and international leaders in understanding the flood risk of individual countries as well as contribute to achieving global targets.

When a catastrophic disaster occurs, the global response generally includes substantial international assistance. A study of the World Bank showed that $69.9 \%$ of international disaster financing is allocated to emergency response while only $3.6 \%$ is allotted for disaster prevention and preparedness (Government of Japan et al. 2012). Although such emergency response saves lives and reduces damage, it does not mitigate risk and prevent future flood events. The SFDRR stresses the importance of understanding and mitigating disaster risks (UNISDR 2015). Quantifying country-based flood risk illustrates the flood risk of each country and identifies priority countries that have high risks and need international assistance before a flood disaster occurs. Country-based quantification at the global scale indicates the issues in need of urgent attention, such as improving coping capacity against a flood. This process can help international organizations, governments, and other stakeholders to examine country-specific conditions and establish and implement policies and strategies toward building a resilient society and supporting sustainable development.

In recent years, several models have been developed for assessing flood risk at regional and global scales (de Moel et al. 2015). Theoretically, risk is computed by multiplying three components: hazard, vulnerability, and exposure (Kron 2005). However, few approaches at the global scale have included all three components and are country-based assessments, owing to difficulties in data availability and vulnerability proxy selection. Hirabayashi et al. (2013) and Arnell and Lloyd-Hughes (2014) developed advanced hydrological models to assess the global impacts of climate change, but their methods were not country-based and did not include vulnerability proxies. Other models have incorporated flood damage as a vulnerability proxy rather than a consequence of a flood disaster. For example, Ward et al. (2013) and Arnell and Gosling (2016) produced global-scale hydrological models with high resolution but did not incorporate the vulnerability component. Dilley et al. (2005) analyzed natural hazard-related disaster hotspots, including flood hotspots at the global scale, but their method did not include exposure and vulnerability. Most other global-scale methods have not appropriately incorporated vulnerability proxies. Meanwhile, the Flood
Vulnerability Index (FVI) comprises meteorological, hydrological, socioeconomic, and countermeasures components (Umemura et al. 2004). The meteorological and hydrological indicators measure hazard, two socioeconomic indicators quantify exposure, and five socioeconomic and two countermeasure indicators represent vulnerability. Therefore, the FVI incorporates the three requisite components of hazard, exposure, and vulnerability. Initially applied to river basins in Japan (Connor and Hiroki 2005), and later, to 114 major river basins across the world (Hara et al. 2009), the index is used only at the riverbasin scale and cannot be used for country-based assessment because of limited data availability. Lee et al. (2015) developed a country-based flood risk method incorporating eight vulnerability proxies and viewed flood risk as expected death tolls due to flood disasters. However, the study covered only 15 Asian countries selected on the basis of data availability. Shi et al. (2016) developed a highresolution global risk index for multiple natural hazards that incorporated hazard, vulnerability, and exposure, and could be applied at the country scale. While the index calculated the flood hazard, the individual vulnerability and exposure components of flooding were not evaluated because they were included as part of multihazard computation.

Limited data availability and vulnerability proxy present major challenges to the development of a method for assessing country-based flood risk. In addition, there is a clear need to include flood management to further improve global-scale methods (de Moel et al. 2015). Therefore, the aim of this study was to develop a method for assessing flood risk assessment that satisfies the following criteria.

(1) Includes the three necessary components of hazard, exposure, and vulnerability

Some previous studies regarded flood loss as flood risk. However, flood damage is a consequence of a flood disaster, and computed risk should appropriately represent the potential damage that is caused by past, present, and future floods. Therefore, this study develops a method that incorporates all three essential components in accordance with risk theory and improves the vulnerability component by including proxies for flood management.

(2) Country-based, global-scale method applicable to most countries in the world

With an aim to contribute toward achievement of global targets such as the SDGs and SFDRR, this study selects and collects data and information that can be applied to most countries at a global scale. Specifically, this study analyzes flood risk at the country scale because countries are the default unit for formulating and implementing international 
targets and national policy as well as for allocating resources.

(3) Improving understanding among leaders and decision makers

The developed method aims to raise awareness among leaders and decision makers, providing easyto-understand guidance and recommending concrete actions for flood risk reduction.

To meet the second and third criteria, a simple formula is developed to address the limited data availability for global coverage that is clear and can be easily understood by leaders and decision makers at the international, national, and local levels.

\section{Methodology}

In this section, the structure of the developed flood risk index that satisfies the three criteria shown in the previous section is described, and the data used to compute the index are explained. The features and limitations of the developed method are also explained.

\subsection{Structure}

Flood risk is determined conceptually by multiplying the three key components: hazard, exposure, and vulnerability (Kron 2005). This study also includes both hard and soft coping capacities that decrease risk in order to factor in flood management. Kannami (2008) also developed an equation including the three key components and the two supplementary components. In that study, hard and soft coping capacities were added together in the denominator of the equation. However, neither hard nor soft coping capacity alone can completely prevent economic damage and human loss, but their combination produces synergistic effects. Therefore, the present study improves the equation used in the earlier study by multiplying the two supplementary components. Thus, the Flood Risk Index (FRI) used in this study is calculated as Eq. 1.

$$
\begin{aligned}
& \text { Flood Risk Index } \\
& \qquad=\frac{\text { Hazard } \times \text { Exposure } \times \text { Basic Vulnerability }}{\text { Hard Coping Capacity } \times \text { Soft Coping Capacity }}
\end{aligned}
$$

The three key components and two supplementary components can be considered as five separate indicators of flood risk, as detailed in Table 1.

Hazard, exposure, and basic vulnerability are the factors related to increased flood risk whereas hard and soft coping capacities are the factors related to decreased risk. The indicators listed in Table 1 are calculated from subindicators that are normalized by expressing them as a percentage
Table 1 Five indicators used to construct the Flood Risk Index

\begin{tabular}{ll}
\hline Indicator & Explanation \\
\hline Hazard & Magnitude of natural phenomena that cause floods \\
Exposure & $\begin{array}{l}\text { Scale of people or areas exposed } \\
\text { Basic } \\
\text { vulnerability }\end{array}$ \\
$\begin{array}{l}\text { Hard coping } \\
\text { capacity }\end{array}$ & $\begin{array}{c}\text { Ability to manage flood disasters through } \\
\text { structural measures }\end{array}$ \\
$\begin{array}{c}\text { Soft coping } \\
\text { capacity }\end{array}$ & $\begin{array}{c}\text { Ability to manage flood disasters through } \\
\text { nonstructural measures }\end{array}$ \\
\hline
\end{tabular}

of the difference between maximum and minimum values, as shown in Eq. 2.

$\operatorname{Subindicator}(i, j)=\frac{x_{i, j}-x_{i, j \min }}{x_{i, j \max }-x_{i, j \min }}$

Here, $i, j, x_{i, j}, x_{i, j \min }$, and $x_{i, j \max }$ are, respectively, indicator number, subindicator number, the subindicator value for a given country, the maximum of the subindicator across all countries, and the minimum of the subindicator across all countries. This unity-based normalization allows for the comparison of values calculated from different datasets at various scales and in various units. Indicators are calculated by summing the subindicators, as in Eq. 3 .

$\operatorname{Indicator}(i)=\sum_{j=1}^{n i} \operatorname{Subindicator}(j)$

In other words, the indicators are calculated using Eq. 3 to sum the constituent subindicators, which are obtained using Eq. 2. The FRI is calculated by inputting the values of these indicators into Eq. 1.

To obtain input data, the Water-Related Disaster Resilience Index (ADB and APWF 2013), World Risk Index (UNU-EHS and Alliance Development Works 2014), Human Development Index (UNDP 2014), Water Poverty Index (Sullivan et al. 2003), and Flood Vulnerability Index (Umemura et al. 2004) were reviewed, and the proxies used for calculating these indices were analyzed. From the viewpoint of data availability and appropriateness, some of the proxies used to calculate those indices were selected for this study, but others were not. For example, population or property in a floodplain and investment amount for river works were not chosen because the global datasets for these items were not available, even though they would be more direct representations of exposure and coping capacity, respectively, compared with the selected proxies. Table 2 shows the list of subindicators that were selected through the process described above and included in this study. 
Table 2 Subindicators of Flood Risk Index

\begin{tabular}{|c|c|c|c|}
\hline Indicator & Subindicator & Data sources, Year & Origin \\
\hline \multirow[t]{6}{*}{ Hazard } & (1) Maximum average monthly precipitation & Weatherbase, 2016 & $\mathrm{E}$ \\
\hline & $\begin{array}{l}\text { (2) Ratio of maximum monthly precipitation to } \\
\text { minimum one }\end{array}$ & Calculation from Weatherbase, 2016 & $\mathrm{E}$ \\
\hline & $\begin{array}{l}\text { (3) Ratio of maximum monthly precipitation to } \\
\text { average annual precipitation }\end{array}$ & Calculation from Weatherbase, 2016 & $\mathrm{E}$ \\
\hline & (4) Maximum average weekly precipitation* & Global Flood Alert System, 2002-2009 & A \\
\hline & (5) Cyclone proneness* & Establishment of Country-Based Flood Risk Index, 1998-2007 & A \\
\hline & $\begin{array}{l}\text { (6) Frequency of over } 100 \mathrm{~mm} \text { precipitation a } \\
\text { day* }\end{array}$ & Global Flood Alert System, 2002-2009 & A \\
\hline \multirow[t]{4}{*}{ Exposure } & (1) Population density & $\begin{array}{l}\text { Population: UN Database, 2015; Land area: SEDAC (NASA), } \\
2016\end{array}$ & A \\
\hline & (2) Urban population growth & UN Database, 2015/1995 & A \\
\hline & (3) Population growth & UN Database, 2015/1995 & A \\
\hline & (4) Ratio of water area to land area & ChartsBin, 2015 & $\mathrm{D}$ \\
\hline \multirow{7}{*}{$\begin{array}{l}\text { Basic } \\
\quad \text { vulnerability }\end{array}$} & (1) Governance (Corruption Perceptions Index) & Transparency International, 2015 & A \\
\hline & $\begin{array}{l}\text { (2) Percentage of population below USD } 1 \\
\text { purchasing power parity (PPP) a day }\end{array}$ & World Development Indicators 2014 (The World Bank) & A \\
\hline & $\begin{array}{l}\text { (3) Net ODA (Official Development Assistance) } \\
\text { of GNI (Gross National Income) (\%) }\end{array}$ & World Development Indicators 2014 (The World Bank) & A \\
\hline & (4) Deforestation rate & $\begin{array}{l}\text { The official United Nations site for the MDGs indicators, } \\
2015 / 2010\end{array}$ & A \\
\hline & (5) Infant mortality rate & CIA World Fact Book, 2016 & A \\
\hline & $\begin{array}{l}\text { (6) Unhealthy life expectancy ( = Total life } \\
\text { expectancy minus healthy life expectancy) }\end{array}$ & Global Health Observatory (GHO) data (WHO), 2016 & $\mathrm{E}$ \\
\hline & (7) Gini Index & CIA World Fact Book & $\mathrm{C}$ \\
\hline \multirow[t]{3}{*}{$\begin{array}{l}\text { Hard coping } \\
\text { capacity }\end{array}$} & $\begin{array}{l}\text { (1) Investment potential density (GDP per land } \\
\text { area) }\end{array}$ & $\begin{array}{l}\text { GDP: Earth trends (World Resources Institute), 2015; Land area: } \\
\text { SEDAC (NASA), } 2016\end{array}$ & A \\
\hline & (2) Total reservoir capacity per land area & $\begin{array}{l}\text { Total reservoir capacity: Global dam reservoir database (Hirano } \\
\text { et al. 2005); Land area: SEDAC (NASA), } 2016\end{array}$ & A \\
\hline & $\begin{array}{l}\text { (3) Proportion of paved roadways to total roadway } \\
\text { length }\end{array}$ & CIA World Fact Book, 2007 & $\mathrm{D}$ \\
\hline \multirow{7}{*}{$\begin{array}{l}\text { Soft coping } \\
\text { capacity }\end{array}$} & (1) Education (Literacy rate) & UN Human Development Report (UNDP), 2014 & A \\
\hline & (2) Education (Gross enrolment ratio) & UN Human Development Report (UNDP), 2014 & A \\
\hline & $\begin{array}{l}\text { (3) Information (Television receivers per } 1000 \\
\text { inhabitants) }\end{array}$ & $\begin{array}{l}\text { Measuring and monitoring the information and knowledge } \\
\text { societies (UNESCO), } 2003\end{array}$ & A \\
\hline & $\begin{array}{l}\text { (4) Information (Mobile cellular telephone } \\
\text { subscriptions per } 100 \text { inhabitants) }\end{array}$ & World Telecommunication/ICT Indicators Database (ITU), 2014 & A \\
\hline & (5) Economy (Gross savings of GDP) (\%) & World Development Indicators 2014 (The World Bank) & A \\
\hline & $\begin{array}{l}\text { (6) Information (Individuals using the Internet of } \\
\text { population) (\%) }\end{array}$ & World Development Indicators 2014 (The World Bank) & $\mathrm{E}$ \\
\hline & (7) Health (Public health expenditure of GDP) (\%) & World Development Indicators 2014 (The World Bank) & $\mathrm{B}$ \\
\hline
\end{tabular}

*These subindicators were used for the analysis of Asia and the Pacific but not for the global analysis due to lack of global-scale data Origins of subindicators: $A$ Water-Related Disaster Resilience Index (ADB and APWF 2013), $B$ World Risk Index (UNU-EHS and Alliance Development Works 2014), $C$ Human Development Index (UNDP 2014), D Flood Vulnerability Index (Umemura et al. 2004), $E$ Originally used in this study

\subsection{Features}

The developed method has the following features.
- Based on risk theory, risk is computed by multiplying the three key components of hazard, vulnerability, and exposure. The developed method improves the inclusion of vulnerability proxies by incorporating basic vulnerability, which represents increased risk, and hard 
and soft capacities, which represent reduced risk. Furthermore, some subindicators of hard and soft capacities are proxies for flood management, which are needed but have rarely been included in assessments of flood risk.

- The risk of flood in most countries, including major population centers, land areas, and economic zones, are assessed and compared in a consistent manner.

- Although policymakers recognize the necessity of improving flood resilience once a catastrophic flood has occurred, their attention is usually focused on other priority issues, such as economic development, before the disaster. Assessing flood risk can help policymakers understand which countries are at most risk as well as illustrate effective measures and strategies that can be implemented before a great flood, making it possible to prepare for flood events and mitigate the impacts in advance.

- Because the method is simple, the results can be presented clearly and their importance can be easily understood by the leaders and decision makers of international organizations, governments, and other stakeholders and subsequently used to formulate development policy and prioritize investment at the global and national scales.

- Each indicator value represents the situations of key components including flood management. Therefore, the results identify challenges and provide guidance on specific issues for more detailed investigation and policy direction to reduce the flood risk.

- Although the method is developed at the global scale, it can be downscaled to national, river basin, and local levels by adjusting data and subindicators to each level.

- A total of 24 subindicators (27 for Asia and the Pacific) are used, with the five indicators calculated by multiple subindicators. Consequently, the developed method includes more extensive factors for assessing flood risk compared with past studies.

- Assessing the flood risk of countries can be further improved by adding or replacing other subindicators when additional global datasets are available.

- Temporal changes in the flood risk of each country can be monitored by using time series data, with changes indicating the effectiveness of measures and policies for flood risk reduction.

The developed method, while an improvement upon earlier methods, nevertheless has some limitations. These are as follows.

- Validation of global flood risk assessments is generally difficult because establishing a meaningful compilation of relevant events with a full geographic spread would require hundreds of years of detailed observation due to the rarity of flood events (de Moel et al. 2015). Although this study uses global datasets spanning 20 years to validate the results, this short duration is insufficient for a complete validation.

- Hazard, exposure, basic vulnerability, and hard and soft coping capacities are equally weighted in this study, and the subindicators used to construct the indicators do not account for the weighting. Assigning a weight to each indicator and subindicator would lead to further improvement.

- In this study, 24 subindicators were used, which is much more than in past studies. However, more directly relevant information, such as the proportions of population and property in floodplains and the investment amounts for river works, was not included, and proxies, such as population density and proportion of paved roadways to total roadway length, were used instead because of the lack of availability of global datasets. With the advancement of technologies and institutional schemes such as information and communications technology and monitoring systems, it is expected that more global datasets will become available and be incorporated into future assessments of flood risk.

\section{Results}

Using the developed method described in the previous section, this study computes and analyzes the flood risks of countries in Asia and the Pacific, which is the most heavily populated part of the world. Home to great geographic, environmental, economic, political, and cultural diversity, the region is prone to severe flooding. Following this regional analysis, the study area is expanded to include the entire world, and country-based flood risks are calculated and evaluated at the global scale.

\subsection{Asia and the Pacific}

Of the 49 member states/regions of the Asian Development Bank (ADB) in Asia and the Pacific, three are excluded due to a general lack of available data (Afghanistan, Bhutan, Myanmar), and another three due to lack of specific data (Brunei, Gini Index; Hong Kong and Taiwan, ratio of water area, proportion of paved roadways, and others). Although some countries/regions are not included, the 33 that are analyzed herein include most of the large countries that account for most of the population, land area, and economic activity of Asia and the Pacific, and so this study is more comprehensive than earlier studies. 


\subsubsection{Regional Analysis}

The FRIs of the 33 countries are computed by the developed method. Figure 1 shows the relationship between FRI and fatalities per 100,000 population caused by floods in each country. The circle size represents the Gross Domestic Product (GDP) per capita of a country. Flood fatalities are calculated using the data between 1996 and 2016 from the Emergency Events Database (CRED n.d.).

Based on the values of flood risk index, the 33 analyzed countries are categorized into low-, medium-, and highflood risk countries, surrounded by green, yellow, and red dotted lines, respectively, in Fig. 1. The figure demonstrates that FRI and flood fatality ratio are related to some extent but are not clearly correlated. In the figure, the location of low-risk countries is limited to the small fatality ratio area whereas some medium-risk states are situated between the small and medium fatality ratio zones. But the high-risk countries range from small to large numbers of fatalities per 100,000 population. This means that a great flood causing a substantial number of fatalities did not occur between 1996 and 2016 in many of the high-risk countries, indicating that a period of 20 years is insufficient for validating a global flood assessment, due to the rarity of great flood events.

The low-risk group includes many developed countries, such as Japan, Australia, and New Zealand, which have low flood risk indices and a small number of deaths per capita. It can be inferred that sufficient investment has been made in flood risk reduction, including infrastructure development supported by their advanced economies. In contrast, high-risk countries such as Cambodia and Nepal experienced a substantial number of fatalities, which are attributable to large floods in 2011 and 2015, respectively. These results suggest that other high-risk countries will incur substantial human losses in the event of a severe flood. However, it is possible for high-risk countries to become medium-risk countries by improving their soft and hard coping capacities and reducing their basic vulnerability and exposure. It is therefore important to grow their economies to enable sustainable investment and improve flood resilience. Some medium-risk countries also have relatively higher fatality ratios and should likewise endeavor to improve their flood resilience.

Another finding is that all the low-risk countries are limited to the small fatality ratio area and none of them have experienced large fatalities per 100,000 population whereas the medium- and high-risk countries occupy a range from small to large fatality ratio areas. This shows that medium- and high-risk countries are vulnerable to floods and incur more fatalities than low-risk counties. Even though the hazard component for some low-risk countries is large and they experience major floods, the number of fatalities is limited because they implemented appropriate flood management and achieved robust flood resilience. In other words, reducing flood risk definitely protects people and saves lives, and so improving flood
Fig. 1 Relationship between Flood Risk Index and fatalities per 100,000 population. Note The green, yellow, and red dotted lines indicate low-, medium-, and high-flood risk country groups, respectively

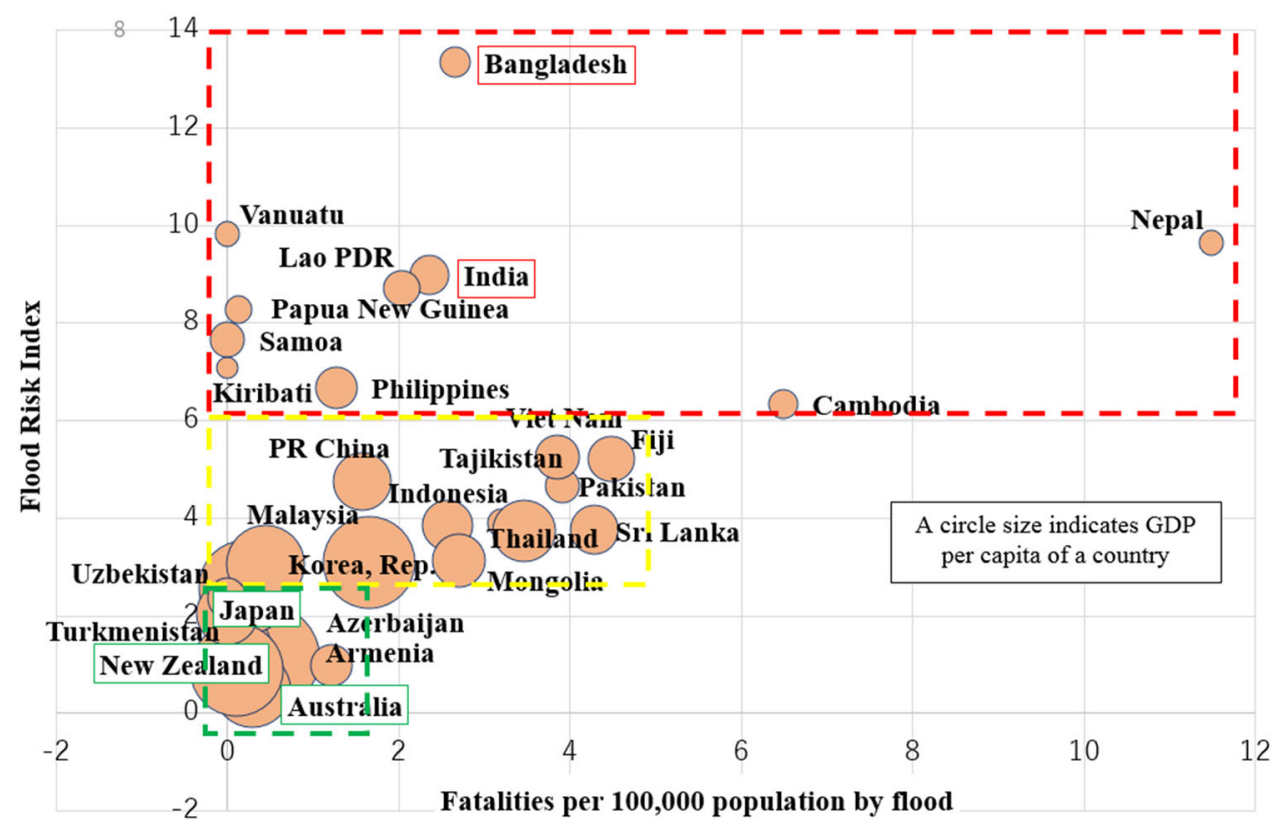


Fig. 2 Composition of Flood Risk Indices in representative high-risk countries (Bangladesh and India) and representative low-risk countries (Japan and Australia)

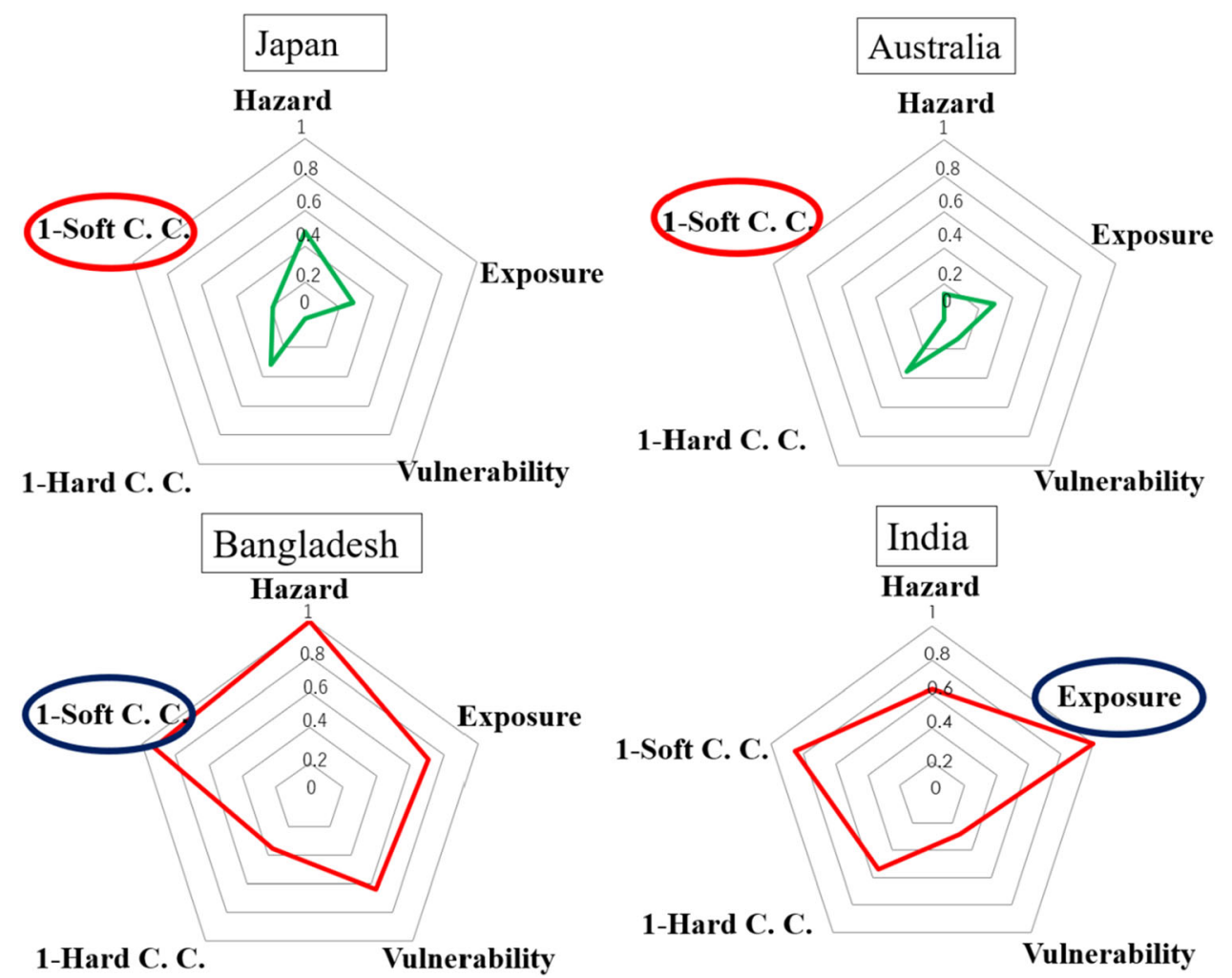

resilience secures people and society regardless of the magnitude and frequency of floods.

\subsubsection{National Analysis}

Figure 2 shows examples of FRI composition in low- and high-risk countries. The five axes in the radar charts represent five indicators (hazard, exposure, basic vulnerability, and hard and soft coping capacities). The values of the five indicators are converted to a range of $0-1$, with larger values for hazard, exposure, and basic vulnerability indicating increased risk and those for hard and soft coping capacities indicating decreased risk. The converted values for hard and soft coping capacities are subtracted from 1 so that larger numbers on all axes of the radar chart indicate increasing risk.

Both Japan and Australia are developed countries and have low FRIs. The charts illustrate that none of the values for either country is high, particularly for soft coping capacity (red). This means that both countries are well prepared for flood disasters because they have implemented appropriate flood management. The values for exposure and basic vulnerability in Japan and Australia are relatively low, which are the result of efforts made by both countries. In terms of hazard, the values for cyclone proneness are not so different between Japan and Australia, but values for precipitation, such as maximum average weekly precipitation and frequency of days with more than $100 \mathrm{~mm}$ of precipitation, are much larger in Japan than in Australia. This reflects the difference in climate between the countries, with Japan having a humid subtropical climate and Australia having an overall dry climate. Although both countries have been improving their flood resilience, Japan still has a higher flood risk than Australia due to its larger hazard, particularly its intensive precipitation.

Bangladesh and India are developing countries situated in South Asia, with large populations concentrated in floodplains, making them vulnerable to flood disasters. Figure 1 shows that both countries are in the high-risk category; Bangladesh has the highest risk among the analyzed countries, although their fatalities per 100,000 population are not so high. Their high flood risks imply that both countries have high potential for extremely high casualties in the event of a great flood. Bangladesh has relatively high exposure because of its high population density and rapid population growth. Its basic vulnerability is also comparatively high due to insufficient governance and economic activity. Bangladesh receives most of its 
precipitation in the rainy season because most land areas are covered by a tropical monsoon climate, and thus the country has the highest possible hazard score. The country's soft coping capacity score is very low (dark blue) due to inadequate education and information systems. The country has dramatically reduced human losses (from around 1000 fatalities in 2004 and 2007 floods to around 100 fatalities in 2012 and 2014 floods), and it is said the improvement in soft coping capacity such as building shelters, community-based flood management, and flood information systems has greatly contributed to the reduction (JICA 2016). Due to the limitation of globally available data, the improvement may not be sufficiently reflected to the developed index, and more detailed study would be needed to clarify the factors of the reduction and show the policy direction for further improvement.

India is also categorized as a high-risk country, but its situation differs from that of Bangladesh. Although its exposure is high (dark blue) and its soft coping capacity is poor, its hazard and basic vulnerability are comparatively lower. India faces the Indian Ocean on both its east and west coasts and is therefore frequently hit by cyclones and its coastal areas receive much precipitation; nevertheless, large parts of the country are situated in dry regions. Consequently, its hazard is not as high as that of Bangladesh. India's basic vulnerability is also not so high compared with Bangladesh because its governance and economic activity are also comparatively more advanced, although still not fully sufficient.

Looking at these four countries, improving resilience is not a priority in Japan and Australia but reducing flood risks in Bangladesh and India is urgent from a regional perspective. Hazard is the magnitude of natural phenomena that cause floods and by definition is something that cannot be managed. Therefore, the priority of Bangladesh should be to improve its soft coping capacity as well as reduce its basic vulnerability and exposure. First, it is recommended that Bangladesh improve its education and information systems to increase its soft coping capacity. Second, while its high population density and rapid population growth increase its exposure, the country can mitigate this by implementing land use plans that identify areas at high risk of flooding and designate restricted zones while relocating vulnerable populations and developing safer areas. Third, it is implied that improved governance and economic systems would decrease basic vulnerability. This result should inform policy goals and as well as indicate what data should be collected and analyzed for building specific strategies.

In India, the priorities are improving soft coping capacity and reducing exposure. However, India is a large country, with a diverse climate, geography, culture, economy, and population. For example, states along major rivers such as the Ganges and Brahmaputra are exposed to high flood risks whereas inland states with a dry climate have a relatively low risk. Therefore, the priorities and measures to be taken for those regions are inherently different. Consequently, further specific studies should be conducted for making concrete policies and strategies that fit the regional features, based on the general guidance provided by this study.

Mapping countries in Asia and the Pacific allows the general relationship between flood risk and fatality ratios to be visualized, revealing three groupings: low, medium, and high risk. Low-risk countries have implemented appropriate disaster risk reduction measures including infrastructure development, which have resulted in decreased fatalities, whereas high-risk countries remain vulnerable to floods and experience substantial loss of human life when great floods occur. This means that improving flood resilience decreases flood risk and secures people and society regardless of the magnitude and frequency of floods. Evaluating the five indicators of an FRI provides important guidance by indicating which issues should be prioritized. This guidance should help international organizations, governments, and other stakeholders to understand the specific factors related to the flood risk of each country and create specific policies and strategies to reduce those risks.

\subsection{Global Scale}

This study expands the target area from Asia and the Pacific to the global scale, and the developed method is applied to 146 of the 193 United Nations member states (47 countries were excluded due to lack of available data). These 146 member states include all major developed countries, such as the Group of Twenty (G20) member states, and represent the vast majority of the global population, land area, and economic zones. Three subindicators (maximum average weekly precipitation, cyclone proneness, and frequency of days with more than $100 \mathrm{~mm}$ precipitation) were not included in the global analysis because of data limitations.

\subsubsection{Global Analysis}

The global map (Fig. 3) below shows the FRIs of 146 countries. Using the values of the FRIs, this study classified the 146 countries into five groups from low to high risk (dark green, light green, yellow, orange, and red). The white areas indicate countries/regions for which data were unavailable.

The map illustrates both regional and country-specific risk and shows that risk tends to be higher closer to the equator. In West Africa, Burkina Faso and Mauritania are at high risk (orange), and Chad, Gambia, Guinea, Liberia, 


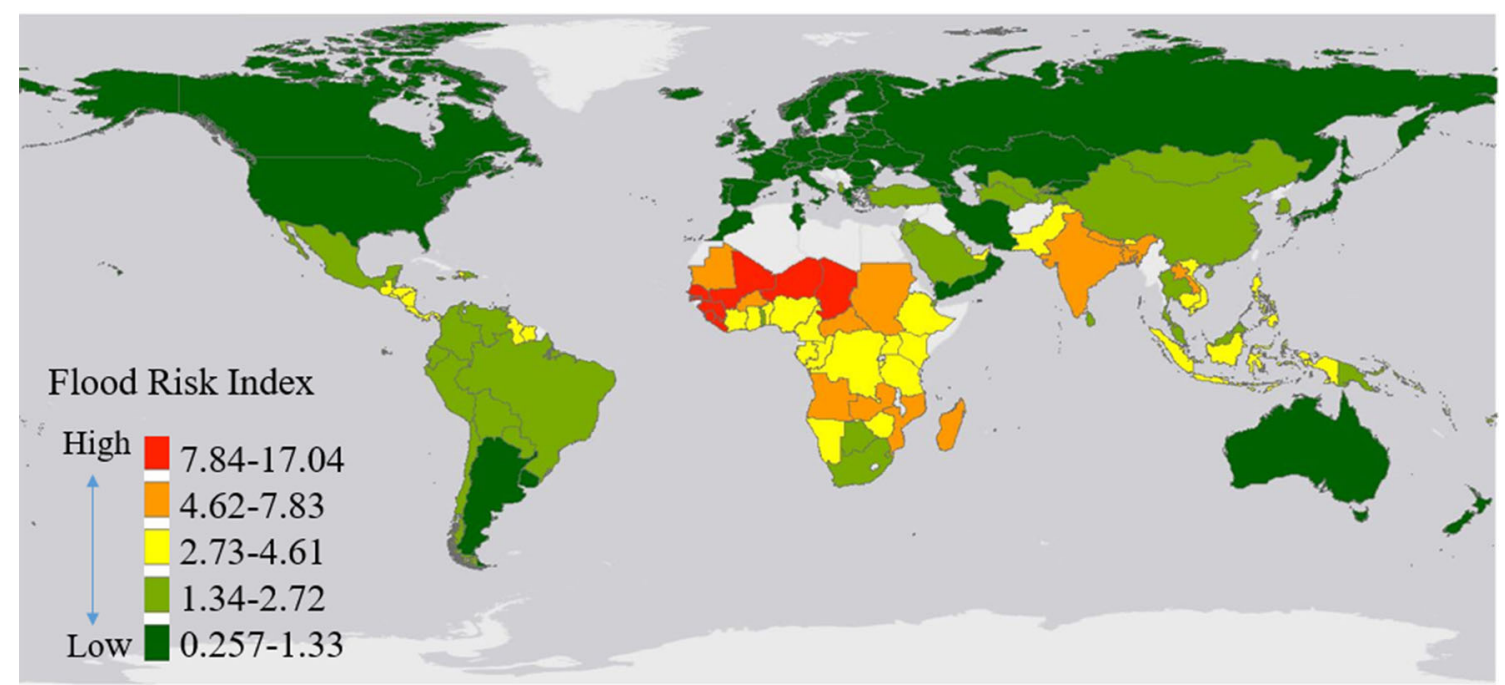

Fig. 3 Map of Flood Risk Index

Mali, Niger, Senegal, and Sierra Leone are at very high risk (red). The countries shown in red all have high hazard and basic vulnerability and low soft coping capacity as well as very low hard coping capacity. Angola, Madagascar, Mozambique, and Zambia in Southern Africa, and Bangladesh, India, Lao People's Democratic Republic (Lao PDR), and Nepal in South and Southeast Asia are also at high risk (orange).

In contrast, developed countries in Europe and North America and in other regions, such as Japan and Australia, have very low risk. Russia and other former Soviet states,
Iran, Oman, and Yemen in the Middle East, Morocco and Tunisia in North Africa, and Argentina in South America are also at very low risk.

To analyze the influences of economic development, which allows for investment in flood management, FRI and GDP per capita are compared, and the result is shown in Fig. 4. The horizontal axis indicates GDP per capita (USD) shown by the common logarithm, and the red dotted line is calculated by the method of least squares. As a general tendency, the larger the per capita GDP, the lower the FRI. In other words, the richer countries are, the more likely
Fig. 4 Relationship between Flood Risk Index and GDP per capita. Note: The red dotted line is the regression line

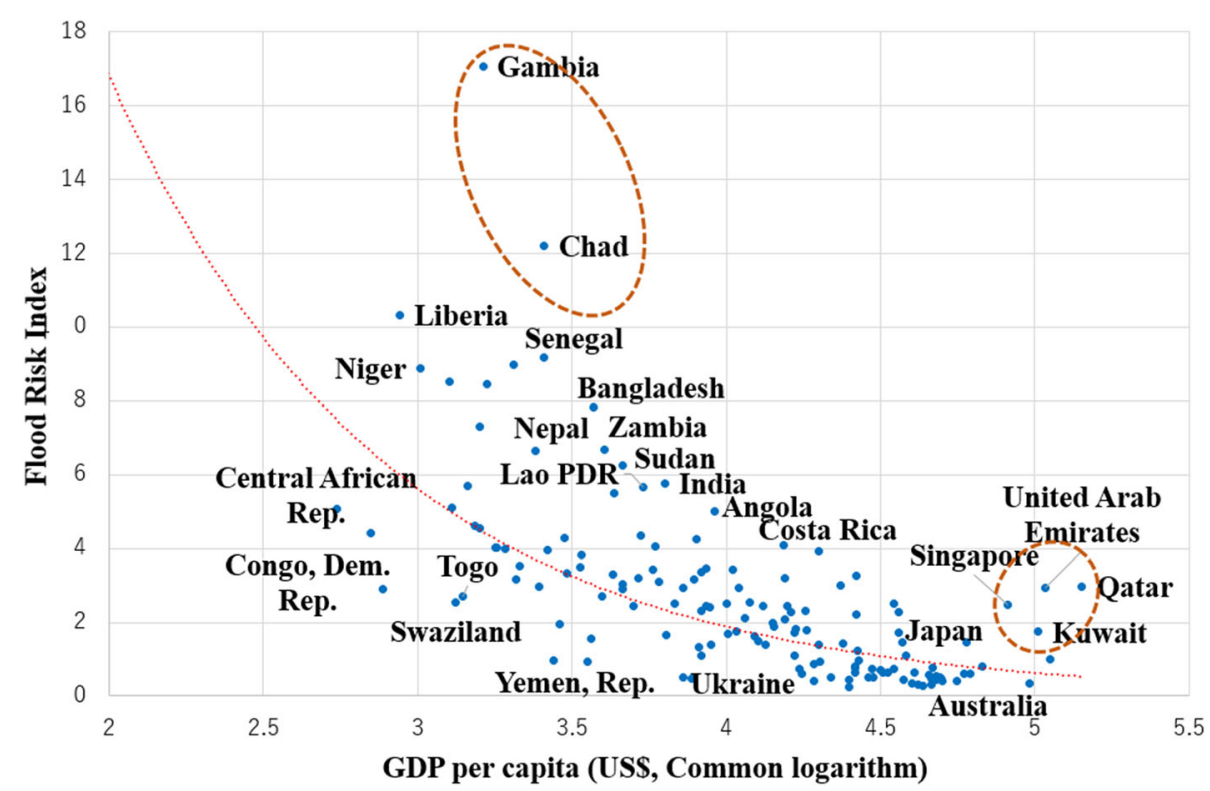


they are to invest in flood risk reduction and keep their risk low.

Gambia has the highest flood risk index of the 146 analyzed countries, followed by Chad, and the index score is higher than those of countries with a similar GDP per capita (brown dashed line). Because developed countries such as Japan, Australia, and EU member states are located in the lower right of the figure, it can be inferred that in these countries per capita GDP is high, flood risk is low, and appropriate investment and countermeasures for flood management are being made.

The countries in the lower right of the figure that are surrounded by the brown dashed line have relatively high FRIs and quite large GDPs per capita. Singapore is an economically growing city-state, and the United Arab Emirates (UAE), Qatar, and Kuwait are all oil-producing countries that are rich in natural resources. In all those countries, their total reservoir capacity per land area in the hard coping capacity is extremely small, which increases their FRIs. Singapore is a small island country with no mountains, and the oil-producing countries rely on groundwater and seawater desalination for their water supply because they are located in dry regions. Therefore, these countries have very little reservoir capacity.

\subsubsection{National Analysis}

Figure 5 shows examples of FRI composition in very highrisk countries (Gambia and Chad) and in medium-risk countries with large GDPs per capita (UAE and Qatar), both types of which are circled by brown dashed lines in Fig. 4.

Gambia has the highest FRI among the 146 analyzed countries, followed by Chad. Gambia is a country with a small land area on the African continent, and due to the effects of explosive population growth and extremely high population density, its exposure is high. Because the literacy rate and school enrollment ratio are low, its soft coping capacity is low, and its hard coping capacity is also low due to extremely inadequate infrastructure investment. Most of its land area has a tropical savanna climate, in which there are distinct wet and dry seasons; that is, most of the annual rainfall occurs during the rainy season, and there is little precipitation during the dry season. Because of the concentrated precipitation in the rainy season, its hazard is very high.

Chad is a large country of more than one million square kilometers, with climates ranging from desert to steppe and savanna. Like Gambia, precipitation is concentrated in the savanna regions, but the overall concentration of rainfall is
Fig. 5 Composition of Flood Risk Indices in high-risk countries (Gambia and Chad) and in medium-risk countries with large GDPs per capita (UAE and Qatar)

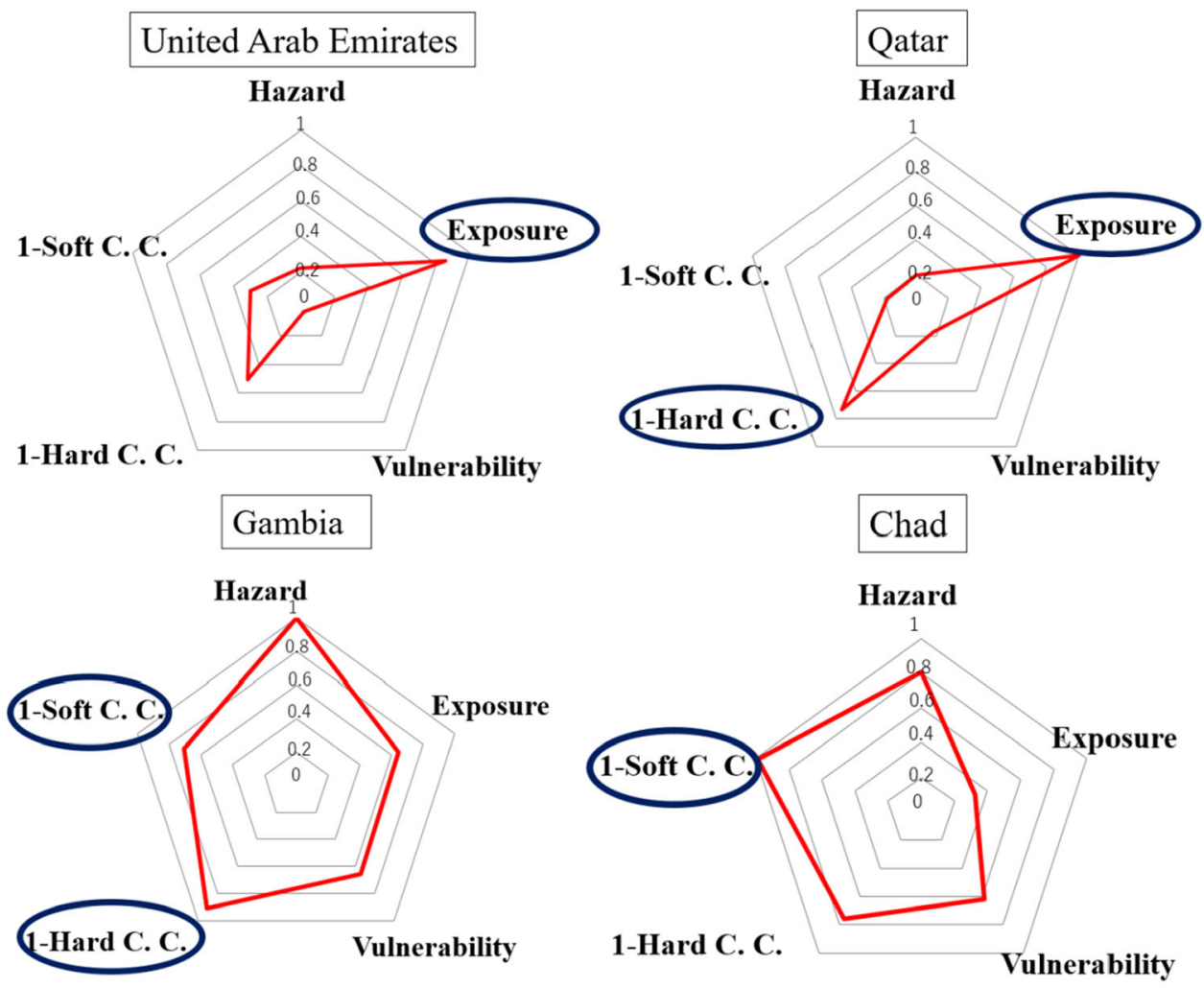


moderate. Therefore, its hazard is not as high as that of Gambia. Due to its sparse population and large land area, its exposure is not high. Because of insufficient infrastructure investment, its hard coping capacity is low. Furthermore, very low literacy rate and school enrollment ratio with deficiencies in other factors give it an extremely low soft coping capacity.

The UAE and Qatar are small countries located on the Arabian Peninsula and have some similarities. Because most of their land area is covered by deserts and rainfall is rare, their hazard is low. Both are oil-rich nations, and thus their economic, educational, and information systems are well developed. Therefore, their basic vulnerability is very low, and their hard and soft coping capacities are high. While their population densities are not high, their populations are concentrated in urban areas and continue to grow at a rapid pace. Consequently, their exposure is very high, which makes both countries high-risk despite their enormous GDPs per capita.

Gambia's fatalities per 100,000 population by flood is 6 and quite large, and that of Chad is 3 and medium compared to those of the countries in Fig. 1. Those losses are the consequences of floods in the past. Figure 4 shows that Gambia and Chad are the countries in most urgent need of reducing their flood risk, even among the least developed countries, because their highest risk means that those countries have most possibilities to suffer large flood disasters in the future. Gambia has distinct wet and dry seasons, most of the population lives in lowland areas along the Gambia River, and these hydrological and geographical conditions put the country at high flood risk. Its insufficient hard and soft coping capacities also contribute to the country's extremely high flood risk. Figure 5 suggests that Gambia's priority should be to grow its hard and soft coping capacities and reduce its exposure. Although a development policy that assists people in relocating from high flood risk zones to safer land areas is needed to decrease exposure, the effectiveness of such a policy would be limited because most of the country's small land area is covered by floodplains. Therefore, it is important for Gambia to increase its soft and hard coping capacities by investing in improvements to its educational, information, health, and infrastructure systems, supported by international institutional and financial assistance.

Similarly, Fig. 5 indicates that Chad should increase its soft and hard coping capacities with international support because the country is also a least-developed state. Unlike Gambia, Chad is a large and sparsely populated country, and thus more region-specific analyses and planning should be prepared. For example, its overall exposure is not so high but this evaluation is based on country-average numbers. Some areas with high flood risk may have a large population, and relocation to safer regions would be an effective option because the country has a vast land area. To formulate a more concrete policy and strategy, a detailed investigation is needed.

Although the UAE and Qatar are considered low-risk countries because they are situated in dry regions, receive little rainfall, and have abundant investment capacity, this study reveals that they are in fact medium-risk countries and should implement appropriate countermeasures. It is true that both countries have low hazard because they receive so little precipitation, but their extremely dense and rapidly growing urban populations put them at a relatively higher risk compared with other similarly wealthy countries. For example, most of the streams in the northern part of the UAE have no hydraulic control structures despite frequent flood events (Elhakeem 2017). This indicates that the government has not adequately invested in flood management, even though it has sufficient financial capacity to do so. Therefore, this study recommends conducting further research with an aim to formulate an appropriate policy and strategy for improving flood resilience.

The global map visualizes regional features and global distribution of low-, medium-, and high-risk countries and provides general guidance to international organizations, governments, and other stakeholders for reducing flood risk. Figure 4 reveals two kinds of high-risk countries at the global scale. One group represents the highest-risk countries among least-developed nations, and the other group includes medium-risk countries with abundant financial resources. Examining the five indicators on a radar chart shows which areas a given country should focus on in formulating policy and strategy to increase its flood resilience, including improvements to educational and information systems and investment in flood defense infrastructure.

\section{Conclusion}

To respond to the international frameworks such as the SDGs and the SFDRR, this study developed a method for assessing flood risk that not only includes its three components, but also enables country-based assessment for the vast majority of countries and can assist leaders and decision makers in understanding flood risk and taking appropriate countermeasures. The developed method is a simple formula that accounts for limited data availability for global coverage, can be easily understood by leaders at the international, national, and local levels, and includes the following features.

- Incorporates the hazard, exposure, and vulnerability components; 
- Is applicable to the vast majority of countries in the world;

- Visualizes priority countries and illustrates effective measures and strategies;

- Is clear and easy to understand by leaders and decision makers of international organizations, governments, and other stakeholders;

- Identifies each country's challenges and provides guidance on specific issues for more detailed investigation and policy creation;

- Includes more extensive factors compared with past studies.

Using the developed method, this study computed the FRIs first of 33 countries in Asia and the Pacific and then of 146 countries in the world. For Asia and the Pacific, the FRI of each country was compared with fatality per 100,000 population caused by floods, and the countries were categorized into low-, medium-, and high-risk. The results show that the medium- and high-risk countries are vulnerable to floods and incur more fatalities compared with low-risk countries. Even though some low-risk countries have large hazards and experience major floods, the number of fatalities is limited because they implemented appropriate flood management and achieved robust flood resilience. In other words, reducing flood risk definitely protects people and saves lives, and so improving flood resilience secures people and society regardless of magnitude and frequency of floods.

This study selected two low-risk countries (Japan and Australia) and two high-risk countries (Bangladesh and India) in Asia and the Pacific and analyzed their flood risks by creating radar charts with five axes that represent the five indicators. The assessment results of these four countries indicate that improving resilience is not a priority for Japan and Australia but reducing flood risk is urgent for Bangladesh and India, and the specific policy direction to each country is shown.

The analysis at the global scale visualizes both regional tendencies and the global distribution of low-, medium-, and high-risk countries. The global map shows that countries closer to the equator tend to have higher flood risk. In contrast, developed countries tend to have lower risk. To examine the influences of economic development, FRI and GDP per capita of 146 countries were compared. As a general tendency, the larger the per capita GDP, the lower the FRI. In other words, the richer countries are, the more likely they are to invest in disaster risk reduction and keep their risk low. The examination also revealed that the two highest-risk countries, Gambia and Chad, were among the least-developed ones. Although the UAE and Qatar might be considered low-risk countries because they are located in dry regions, receive little rainfall, and have abundant investment capacity, this study revealed that they are in fact medium-risk countries. This study gave the specific policy direction to each country.

Evaluating the five indicators of an FRI provides important guidance by indicating which issues should be prioritized.

The developed method improved upon earlier methods, but further improvements can be made. One is to validate flood risk assessments by using sufficient amounts of data. Another is to add weight to indicators and subindicators. The last is to incorporate more directly relevant information for subindicators, which should be facilitated by further advancements in information and communications technology and institutional monitoring systems.

Acknowledgements I would like to thank the colleagues at Yamaguchi University for their supports in conducting this research. I would also like to thank Dr. Syota Sasaki and Mr. Kento Fujioka for their contribution to the preliminary work of this study, and Prof. Miho Ohara for her valuable comments in improving the study.

Open Access This article is licensed under a Creative Commons Attribution 4.0 International License, which permits use, sharing, adaptation, distribution and reproduction in any medium or format, as long as you give appropriate credit to the original author(s) and the source, provide a link to the Creative Commons licence, and indicate if changes were made. The images or other third party material in this article are included in the article's Creative Commons licence, unless indicated otherwise in a credit line to the material. If material is not included in the article's Creative Commons licence and your intended use is not permitted by statutory regulation or exceeds the permitted use, you will need to obtain permission directly from the copyright holder. To view a copy of this licence, visit http://creativecommons. org/licenses/by/4.0/.

\section{References}

ADB (Asia Development Bank), and APWF (Asia-Pacific Water Forum). 2013. Asian water development outlook 2013. Metro Manila: ADB.

Arnell, N.W., and S.N. Gosling. 2016. The impacts of climate change on river flood risk at the global scale. Climate Change 134: 387-401.

Arnell, N.W., and B. Lloyd-Hughes. 2014. The global-scale impacts of climate change on water resources and flooding under new climate and socio-economic scenarios. Climate Change 122: 127-140.

Connor, R.F., and K. Hiroki. 2005. Development of a method for assessing flood vulnerability. Water Science \& Technology 51(5): 61-67.

CRED (Centre for Research on the Epidemiology of Disasters). n.d. Emergency Events Database (EM-DAT). https://www.emdat.be/. Accessed 2 May 2018.

CRED (Centre for Research on the Epidemiology of Disasters), and UNISDR (United Nations International Strategy for Disaster Reduction). 2015. Weather related disasters 1995-2015. In The human cost of weather related disasters 1995-2015, 7-11. Brussels and Geneva: CRED and UNISDR. 
de Moel, H., B. Jongman, H. Kreibich, and E.C. Penning-Rowsell. 2015. Flood risk assessment at different spatial scales. Mitigation and Adaptation Strategies for Global Change 20: 865-890.

Dilley, M., R.S. Chen, U. Deichmann, A.L. Lerner-Lam, M. Arnold, J. Agwe, P. Buys, O. Kjekstad, et al. 2005. Natural disaster hotspots. The World Bank Disaster Risk Management Series. Washington, DC: World Bank.

Elhakeem, M. 2017. Flood prediction at the northern region of UAE. MATEC Web of Conferences 103: Article 04004.

Government of Japan, GFDRR (Global Facility for Disaster Reduction and Recovery), and the World Bank. 2012. The Sendai report: Managing disaster risks for a resilient future. Washington, DC: World Bank.

Hara, Y., K. Umemura, K. Kato, R.F. Connor, and Y. Sato. 2009. The development of flood vulnerability index applied to 114 major river basins around the world. Journal of Japan Society of Hydrology and Water Resources 22(1): 10-23.

Hirabayashi, Y., R. Mahendran, S. Koirala, L. Konoshima, D. Yamazaki, S. Watanabe, H. Kim, and S. Kanae. 2013. Global flood risk under climate change. Nature Climate Change 3: 816-821.

Hirano, J., J. Magome, H. Ishidaira, and K. Takeuchi. 2005. Global dam reservoir database for large scale hydrological analysis. Annual Journal of Hydraulic Engineering 49: 385-390.

JICA (Japan International Cooperation Agency). 2016. Detailed planning study report: The Project for Research on Disaster Prevention/Mitigation Measures against Floods and Storm Surges in Bangladesh. Tokyo: JICA.

Kannami, Y. 2008. Establishment of country-based flood risk index. Tokyo and Ibaraki-ken, Japan: National Graduate Institute for Policy Studies (GRIPS) and International Centre for Water Hazard and Risk Management (ICAHRM).

Kron, W. 2005. Flood risk = hazard exposure vulnerability. Water International 30(1): 58-68.
Lee, S., T. Okazumi, Y. Kwak, and K. Takeuchi. 2015. Vulnerability proxy selection and risk calculation formula for global flood risk assessment: A preliminary study. Water Policy 17: 8-25.

Shi, P., X. Yang, W. Xu, and J. Wang. 2016. Mapping global mortality and affected population risks for multiple natural hazards. International Journal of Disaster Risk Science 7(1): $54-62$.

Sullivan, C.A., J.R. Meigh, A.M. Giacomello, T. Fediw, P. Lawrence, M. Samad, S. Mlote, and C. Hutton et al. 2003. The water poverty index: Development and application on the community scale. Natural Resource Forum 27: 189-199.

Umemura, K., K. Hiroki, K. Kato, K. Hashimoto, Y. Hara, and T. Ishio. 2004. Formation of Flood Vulnerability Index (FVI): Preliminary computation of FVI. In Proceedings of the Annual Conference of Japan Society of Civil Engineers 2(057): 113-114.

UNDP (United Nations Development Programme). 2014. Human development report 2014. New York: UNDP.

UNISDR (United Nations International Strategy for Disaster Reduction). 2007. Hyogo framework for action 2005-2015. Geneva: UNISDR.

UNISDR (United Nations International Strategy for Disaster Reduction). 2015. Sendai framework for disaster risk reduction 2015-2030. Geneva: UNISDR.

United Nations. 2015. Transforming our world: The 2030 agenda for sustainable development. New York: United Nations.

UNU-EHS (United Nations University-Institute for Environment and Human Security), and Alliance Development Works. 2014. World risk report 2014. Bonn and Berlin: UNU-EHS and Alliance Development Works.

Ward, P.J., B. Jongman, F.S. Weiland, A. Bouwman, R. Beek, M. Bierkens, W. Ligtvoet, and H. Winsemius. 2013. Assessing flood risk at the global scale: Model setup, results, and sensitivity. Environmental Research Letters 8: 1-10. 